\title{
A Mobile Multicast Framework for CDMA-based Ad Hoc Networks
}

\author{
Hsu-Yang Kung and Su-Man Chen \\ Department of Management Information Systems \\ National Pingtung University of Science and Technology, Pingtung, Taiwan 91207
}

\begin{abstract}
The objective of this paper is (i) to construct a robust ad hoc mobile network using the Direct Sequence CDMA technology, (ii) to self-create the transmission schedules for downlink and uplink communications, and then (iii) to multicast data packets with the collision-free transmission and the hidden-terminal avoidance. In this paper, we proposed the Hierarchical Linked Cluster Multicast (HLCM) network architecture and the HLCM Network Formation algorithm based on the characteristic of the DS-CDMA communication to construct a robust ad hoc multicast network. To achieve the collision-free and hidden-terminal avoidance multicast communications, the Uplink Multicast Schedule Algorithm (UMSA) and the Downlink Multicast Schedule Algorithm (DMSA) are proposed to generate the intra-cluster and inter-cluster transmission schedules, respectively. Each mobile node multicasts data packets to the destination nodes according to the pre-assigned time slots of the transmission schedules. The corresponding simulation results show that the proposed algorithms and control schemes effectively solve the hiddenterminal problem and achieve the self-organization and self-operation of a CDMA-based ad hoc multicast network.
\end{abstract}

Key words: Ad Hoc Network, CDMA, Hierarchical Linked Cluster, Hidden Terminal Problem, Multicast Communications.

\section{INTRODUCTION}

As the rapid progress of broadband wireless/mobile network technology, the state-of-the-art mobile multimedia applications, such as the Mobile Video Conferencing and Mobile Telemedicine, are eagerly required and developed $[1,2,11]$. For such popular mobile multimedia applications, many of them are based on an ad hoc network. There are two essential technology issues, which are (i) the creation and operation of an ad hoc network among the mobile hosts/devices to quickly adapt the mobility and The original version of this chapter was revised: The copyright line was incorrect. This has been
corrected. The Erratum to this chapter is available at DOI: $10.1007 / 978-0-387-35618-1 \_37$ 
(ii) the provision of the multicast communications to effectively reduce the consumption of the wireless network bandwidth $[3,4,6]$. Ad hoc wireless networks consist of mobile hosts and dynamically create a communication network among them without using any infrastructure or administrative devices [14]. In contrast to conventional wireless networks, which require the provisions of the prerequisite network infrastructure and centralized administration devices for mobile communications, ad hoc wireless networks can adapt the dynamic network topology to the mobile hosts and are self-creation, self-organization, and self-administration with the collision-free and hidden-terminal avoidance $[3,7,12,13,15]$. The "hiddenterminal" problem in an ad hoc network is that two mobile nodes simultaneously transmit messages to the third node and the transmission collision occurs. The third node is the "hidden-terminal".

The Third-Generation (3G) wireless network, which provides higher bandwidth and could achieve mobile multimedia applications, is the key revolution of the broadband wireless/mobile network $[5,8]$. The air interface of $3 \mathrm{G}$ focuses on the Code Division Multiple Access (CDMA) technology, which supports a high transmission rate and has the characteristics, including the anti-jamming, high capacity, and security, is very suitable for multimedia communications in ad hoc networks $[6,9,10]$. For example, each mobile device of a Mobile Video Conferencing, which is a CDMA-based communication system, can dynamically join or leave the conference and achieve perceivable presentation qualities with feasible communication schemes. In such kinds of mobile multimedia applications, it is critically required to rapidly construct an ad hoc mobile network with a robust topology, and distribute the data packets with collision-free multicasting [14, $15,16]$. There are few researches on the self-organization and self-operation of a feasible CDMA-based ad hoc network with the provision of multicast communications. To achieve the characteristics of ad hoc multicast networks, the state-of-the-art organization and communication schemes on the CDMA air interface are urgently required. In this paper, we proposed (i) the Hierarchical Linked Cluster Multicast (HLCM) network architecture, which is a robust ad hoc mobile network using the Direct Sequence CDMA (DS-CDMA) technology, and (ii) the transmission schedules with selfcreation for downlink and uplink multicast communications with the collision-free transmission and the hidden-terminal avoidance.

The organization of the rest of this paper is as follows. In Section 2, we introduce the HLCM network infrastructure. Section 3 describes the proposed construction and multicast schedule algorithm. Section 4 describes the simulation results of the proposed algorithms. Section 5 presents our conclusion. 


\section{HIERARCHICAL LINKED CLUSTER MULTICAST NETWORK INFRASTRUCTURE}

The proposed Hierarchical Linked Cluster Multicast (HLCM) network architecture is based on the characteristic of the DS-CDMA communication. As depicting in Figure 1, the whole CDMA frequency spectrum is divided into $M$ frequency sub-bands, each of which conducts a communication range and forms a specific network topology. Each sub-band is composed of two communication periods, which are (i) the epoch period and (ii) the data communication period. An epoch period is the time period of creating and organizing an ad hoc mobile network. During each sub-band, the epoch period repeats periodically to ensure an up-to-date linkage of the network. A data communication period is the time period that all mobile hosts multicast data packets according to the transmission schedules for the specific $H L C M$ ad hoc network.

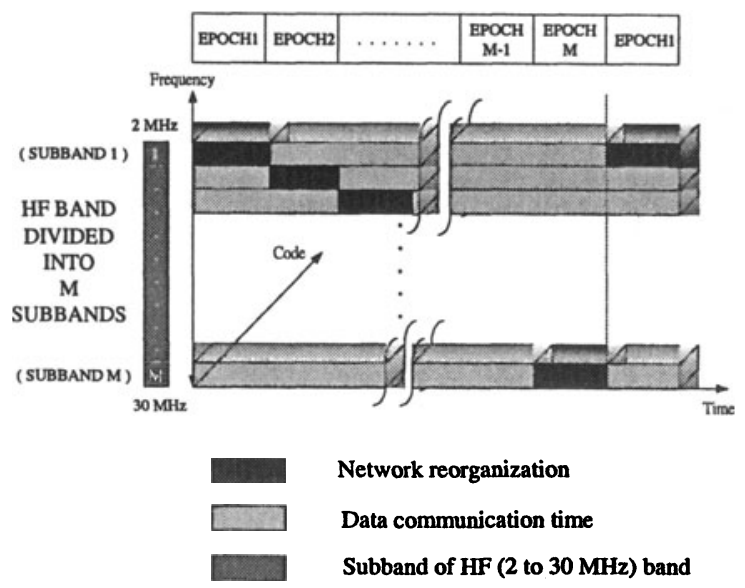

Figure 1. The frequency, time, and code division structure for the organization and communication of the HLCM network.

Figure 2 depicts a $H L C M$ network topology, which is composed of hierarchical linked clusters. Mobile nodes in a HLCM cluster are defined as follows.

(1) Cluster Head. Each cluster has only a cluster head, which is the based station (BS) in a cluster. For example, the nodes $H_{1}, H_{3}, H_{4}$, and $H_{9}$ are the cluster heads of clusters $C_{1}, C_{3}, C_{4}$, and $C_{9}$, respectively.

(2) Gateway Node. It is an interconnection node, which is responsible for forwarding data packets between/among clusters. To construct a robust ad hoc network, three kinds of gateway nodes are specifically defined. (i) The Overlapping Gateway. It is located in the overlapping area between clusters. (ii) The Non-overlapping Gateway. If two clusters don't overlap and have at least a pair of nodes, each of which is located 
in the corresponding cluster and can communicate with each other. These two nodes are the non-overlapping gateways. (iii) The Duality Gateway. A node not only is an overlapping gateway but also is a nonoverlapping gateway.

(3) Ordinary Node. An ordinary node is neither the cluster head nor the gateway.

\section{SYSTEM IMPLEMENTATION}

In this Section, we proposed three algorithms to achieve the selfconstruction of the HLCM network and the uplink/downlink multicast communications with collision-avoidance. To achieve the network selforganization and self-linkage, we proposed an HLCM Network Construction (HLCM-NC) algorithm, which operates in the epoch period, to rapidly construct a two-tier hierarchical ad hoc network. Figure 3 depicts the structure of the epoch period. The HLCM-NC algorithm divides each epoch into two frames and some time slots. Two frames $F_{1}$ and $F_{2}$ are further divided $N$ time slots and $N$ is the number of all nodes in the ad hoc network. Each node broadcasts the control message in its assigned time slot of the two frames according to the node ID. The transmission order in frame $F_{1}$ is with the increased manner, i.e., from node $N_{l}$ to node $N_{N}$, and the

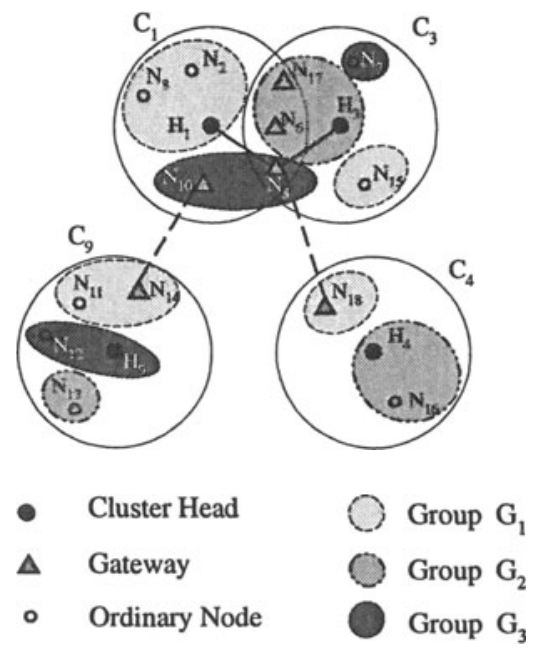

Figure 2. A HLCM network topology.

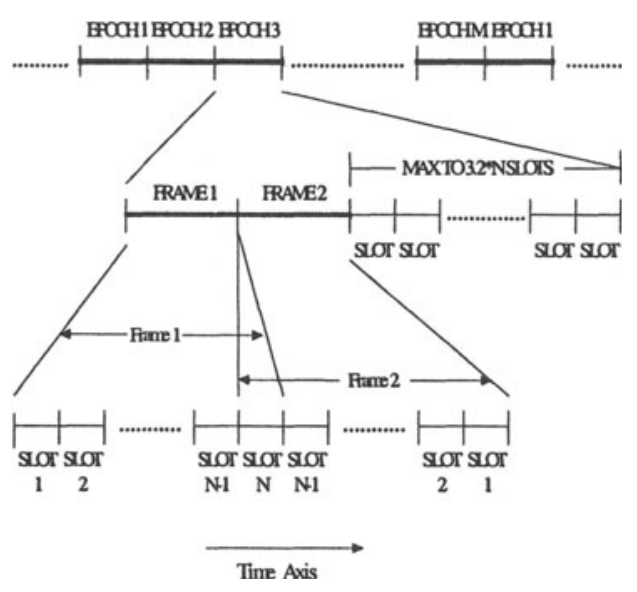

Figure 3. The structure of the epoch period.

transmission order in frame $F_{2}$ is with the decreased manner, i.e., from node $N_{N}$ to node $N_{l}$.

The construction operations during frame $F_{1}$ are as follows. (i) The node $N_{H}$, which doesn't "hear" any node announce to be a cluster head 
before $N_{H}$ 's announcement, $N_{H}$ becomes a cluster head and broadcasts this message in its assigned time slot. (ii) A node $N_{i}$, which "hears" the node $N_{H}$ announce to be a cluster head before its announcement, the node $N_{i}$ becomes a member of the cluster $C_{H}$ and broadcasts its node ID. (iii) A node $N_{g}$, which "hears" two or more than two nodes announce to be cluster heads before $N_{g}$ 's announcement, $N_{g}$ becomes an overlapping gateway and broadcasts this message in its assigned time slot. (iv) A node $N_{x}$, which "hears" a node $N_{H}$ announce to be a cluster head and the other node $N_{y}$ announce to be a member of another cluster before $N_{x}$ 's announcement, $N_{x}$ pairs for $N_{y}$ and becomes the non-overlapping gateway. $N_{x}$ broadcasts this message in its assigned time slot. (v) A node $N_{d}$, which already announced to be an overlapping gateway and then "hears" the other node $N_{y}$ announce to be a member of another cluster, $N_{d}$ becomes a duality gateway. (iv) If a node $N_{j}$ joins a multicast group $\mathrm{G}_{\mathrm{k}}, N_{j}$ announces its multicast group $\mathrm{ID}\left(G_{k}\right)$ in its broadcasting time slot.

After frame $F_{1}$ of the epoch period, we have the following realization. (i) Each cluster head realizes its cluster members. (ii) Each overlapping gateway realizes its cluster head. (iii) Each non-overlapping gateway announces its cluster heads and the linked clusters. However, the cluster head doesn't agree yet. (iv) Each ordinary node realizes its cluster head. (v) Each cluster head realizes the multicast groups in its cluster.

The construction operations during frame $F_{2}$ are as fellows. (i) Each cluster head announces its members, the multicast groups IDs, neighbouring cluster heads, and the gateways including the overlapping and nonoverlapping gateways. (ii) Each overlapping gateway announces its cluster heads and the linked clusters. (iii) Each non-overlapping gateway announces its cluster head and the linked clusters after selecting by the cluster head. (iv) Each ordinary node announces its cluster head. (v) Each cluster head realizes the groups in the neighbouring clusters.

To achieve the collision-free and hidden-terminal avoidance multicast communication, we proposed the Uplink Multicast Schedule Algorithm (UMSA) and the Downlink Multicast Schedule Algorithm (DMSA), which construct the Intra-Cluster and the Inter-Cluster transmission schedules, respectively. To achieve message routing for building transmission schedules, there are four kinds of cluster heads are specified: the Start Head (SH), the Mediate Head (MH), the End Head (EH), and the Isolated Head (IH). Between/among the neighbouring clusters, the smallest (largest) ID cluster heads are the SHs (EHs), and the other cluster heads are MH. The IHs have no neighboring cluster head. Based on the transmission of SHs, MHs, and EHs, the multicast schedules for uplink and downlink communications are self-created.

Based on the UMSA and DMSA, each cluster head creates the uplink and downlink multicast schedules and broadcasts the schedules to all nodes during the epoch period. Using the uplink multicast schedule, each node of 
the cluster can transmit data packets to its head in the pre-assigned time slots with collision-avoidance during the data communication period. Using the downlink multicast schedule, each cluster head can multicast data packets to all members of a multicast group between clusters via gateway nodes. Based on the transmission schedules, the hidden-terminal problem is effectively solved.

\section{SIMULATION RESULTS}

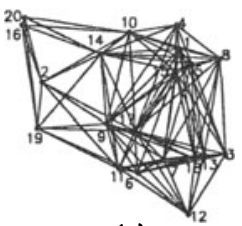

(a)

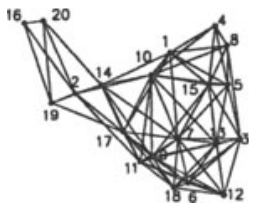

(c)

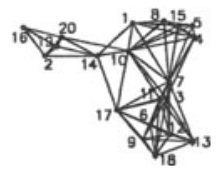

(e)

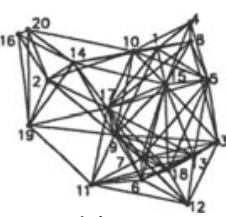

(b)

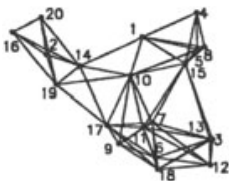

(d)

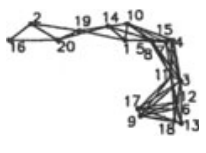

(f)

Figure 4. An example of the connection topologies based on six different radio frequencies.

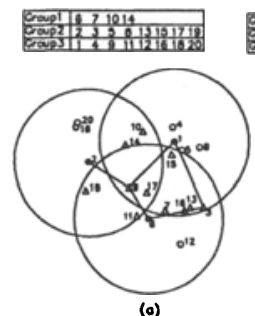

(o)

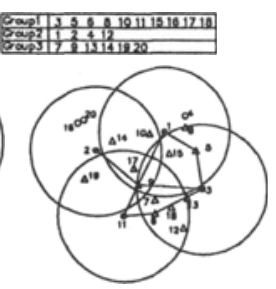

(b)

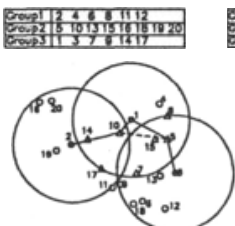

(c)

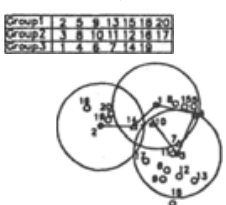

(e)

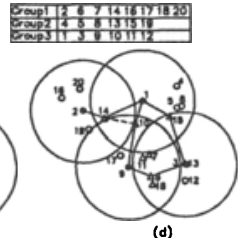

(d)

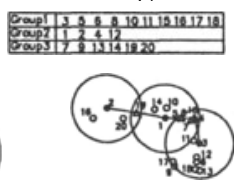

(f)

Figure 5. The corresponding HLCM network topologies based on Figure 4.

As depicting in Figure 4, the simulation models are composed of 20 mobile hosts, which form different connection topologies and have different mobility capabilities based on six different radio frequencies. Each mobile host is initially positioned randomly and free to move. By applying the proposed HLCM Network Construction algorithm, a robust ad hoc network is self-created and self-organized among mobile hosts. The corresponding $H L C M$ networks based on different connection topologies are illustrated in Figure 5.

Tables 1 and 2 show the uplink and downlink multicast schedules corresponding to the examples of Figure 5 based on the UMSA and DMSA 
schemes. In the uplink multicast schedule, the "*” represents the start head, the "\#" represents the end head, the " + " represents the isolated head, and the mediate node is represented with blank. Each cluster member is assigned unique time slots for transmitting data packets to its head to ensure collisionfree and hidden-terminal avoidance. In the downlink multicast schedule, each cluster head assigns dedicated time slots for each of its gateways to avoid collision. The cycle length of the transmission schedule is the sum of the time slots that the related cluster heads arrange for a specific gateway.

Table 1. The uplink multicast schedule corresponding to Figure 5.

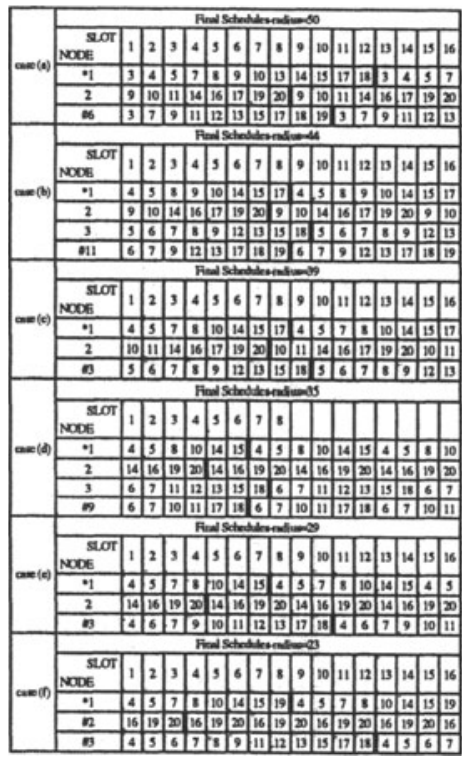

Table 2. The downlink multicast schedule corresponding to Figure 5.
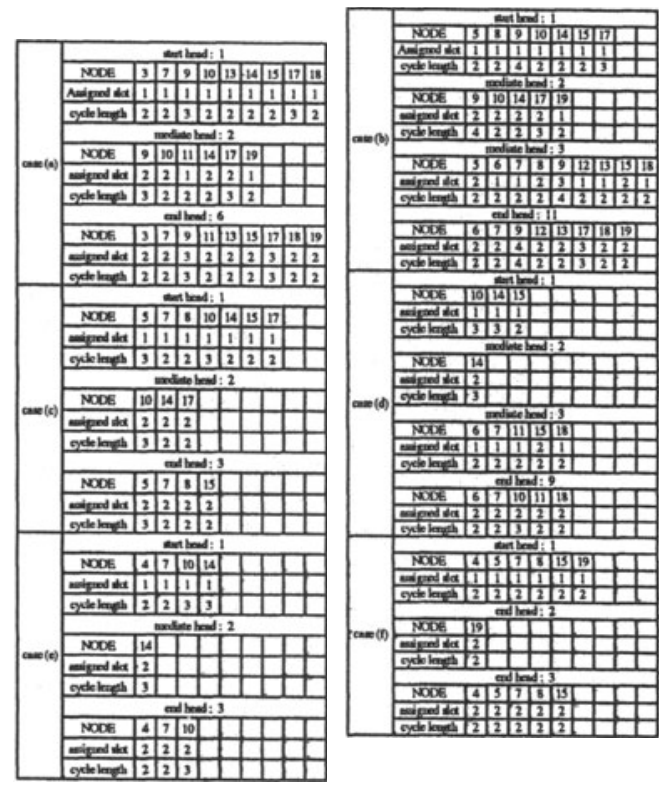

\section{CONCLUSION}

In this paper, we proposed the Hierarchical Linked Cluster Multicast (HLCM) network architecture and the HLCM Network Construction algorithm based on the characteristic of the DS-CDMA communication to construct a robust ad hoc multicast network. To achieve the collision-free and hidden-terminal avoidance multicast communications, the Uplink Multicast Schedule Algorithm (UMSA) and the Downlink Multicast Schedule Algorithm (DMSA) are proposed to generate the intra-cluster and intercluster transmission schedules, respectively. Each mobile node multicasts data packets to the destination nodes according to the pre-assigned time slots 
of the transmission schedules. The simulation results show that the proposed algorithms and control schemes effectively solve the hidden-terminal problem and achieve the self-organization and self-operation of a CDMAbased ad hoc multicast network.

\section{References}

[1] F. Adachi, M. Sawahashi, and H. Suda, "Wideband DS-CDMA for next-generation mobile communications systems," IEEE Communications Magazine, Vol. 36, No. 9, pp. $56-69$, Sept. 1998.

[2] C. P. Blum, R. Dubois, R. Molva, and O. Schaller, "A development and runtime platform for teleconferencing applications," IEEE Journal on Selected Areas in Communications, Vol. 15, No. 3, pp. 576-588, Apr. 1997.

[3] S. Chakrabarti and A. Mishra, "QoS Issues in Ad Hoc Wireless Networks", IEEE Communications Magazine, Vol. 39, Issue 2, pp. 142 -148, Feb. 2001

[4] Y. Chun, X.L. Ming, and S. .M. Lin, "Hierarchical On-demand Routing for SelfOrganized Networks," Fifth Asia-Pacific Conference on Communications, Vol. 1, pp. 128-133, 1999.

[5] C. Comaniciu, N. B. Mandayam, D. Famolari and P. Agrawal, "QoS Guarantees for Third Generation (3G) CDMA Systems via Admission and Flow Control," Proceedings of IEEE VTC'00- Fall, Sep. 2000, pp. 249-256.

[6] N. Dimitriou, R. Tafazolli, and G. Sfikas, "Quality of service for multimedia CDMA," IEEE Communications Magazine, Vol. 38, No. 7, pp. 88-94, July 2000.

[7] I. Gupta, "Minimal CDMA Recoding Strategies in Power-controlled Ad-hoc Wireless Networks", Proc. 1st International Workshop on Parallel and Distributed Computing Issues in Wireless Networks and Mobile Computing, April, 2001.

[8] O. Gurbuz and H. Owen, "Dynamic Resource Scheduling Schemes for W-CDMA Systems," IEEE Communications Magazine, Vol. 38, No. 10, Oct. 2000, pp. 80-84.

[9] Y. Han and H. G. Bahk, "CDMA Technology: Present Status and Future Prospects," Asia Pacific Microwave Conference, pp. 165-168, 1997

[10] P. M. Mistry, "Third Generation Cellular (3G): W-CDMA \& TD-CDMA," Wescon/98, pp. 227-231, 1998.

[11] M. W. Oliphant, "The Mobile Phone Meets the Internet," IEEE Spectrum, Vol. 36, No. 8, pp. 20-28, Aug. 1999.

[12] G. Pei, M. Gerla, M., X. Hong, and C. C. Chiang, "A Wireless Hierarchical Routing Protocol with Group Mobility," Wireless Communications and Networking Conference (WCNC), Vol. 3, pp. $1538-1542,1999$.

[13] C. E. Perkins, "Mobile Networking in the Internet," Mobile Networks and Applications, Vol. 3, 1998, pp 319-334.

[14] R. Ramanathan and M. Steenstrup, "Hierarchically-organized, multihop mobile wireless networks for quality-of-service support," Mobile Networks and Applications, Vol. 3, No. 1, June 1998, pp. 101-119.

[15] J. Ryu, S. Song, and D. H. Cho, "A Power-Saving Multicast Routing Scheme in 2-tier Hierarchical Mobile Ad-Hoc Networks," Vehicular Technology Conference(VTC), Vol. 4, pp. $1974-1978,2000$.

[16] M. O. Sunay, S. Tekinay, and S. Z. Ozer, "Efficient allocation of radio resources for CDMA based wireless packet data systems," Global Telecommunications Conference (GLOBECOM), Vol. 1b, pp. 638 -643, 1999. 